\title{
Anxiety and failure in concept identification
}

\author{
KAREN MEITES and VLADIMIR PISHKIN \\ Oklahoma City Veterans Administration Medical Center, Oklahoma City, Oklahoma 73104, and \\ University of Oklahoma Health Sciences Center, Oklahoma City, Oklahoma 73069
}

and

LYLE E. BOURNE, JR.

Department of Psychology and Institute of Cognitive Science

University of Colorado, Boulder, Colorado 80302

\begin{abstract}
This study compared 56 undergraduates exhibiting high or low anxiety on their ability to solve a concept identification problem following success or failure on a prior problem. Those low in anxiety showed better performance on the problem following failure; those high in anxiety showed better performance following success. This outcome is consistent with previously reported studies using patients hospitalized for anxiety reactions, but it differs from those comparing Type A and Type B coronary-prone personalities.
\end{abstract}

Pishkin (1965) found that antecedent experience with an insoluble problem creates a realistic experience of stress and failure in normal adult subjects and consequently inhibits their performance on a concept identification (CI) task relative to subjects who succeed on or are given no antecedent problem. Because of the implications of failure (stress) and success for certain clinical groups, this paradigm was administered subsequently to hospitalized anxiety reaction patients (Pishkin, Fishkin, Shurley, Lawrence, \& Lovallo, 1978) and to Type A and B coronary-prone individuals (Lovallo \& Pishkin, 1980), with somewhat conflicting results. With $\mathrm{A} / \mathrm{B}$ types, it was demonstrated that performance differences attributable to prior success vs. failure obtain only for Type B subjects. With anxiety reaction patients taking an antidepressant medication, antedecent failure inhibits CI performance as it does with normals, although the effect is exaggerated. The patient data were further analyzed after dividing subjects at the median of their scores on the Manifest Anxiety Scale (MAS) (Taylor, 1956). Patients above the median in anxiety who failed the first problem were superior to all other groups on the second problem.

Thus, anxiety (in patients) potentiated performance differences within the success/failure CI paradigm,

The analysis of variance was conducted using BMDPV2, 1977 series, developed at the Health Sciences Computing Facility, University of California, Los Angeles, under NIH Special Resources Grant RB-3. The authors wish to express their appreciation to W. R. Lovallo and R. A. Yaroush for their valuable assistance and advice in this study. This study was conducted while Lyle E. Bourne, Jr., was on sabbatical semester at the Department of Psychiatry and Behavioral Sciences of the University of Oklahoma College of Medicine, Oklahoma City. Send reprint requests to Vladimir Pishkin (151A), Behavioral Sciences Laboratories, Veterans Administration Medical Center, 921 N.E. 13th Street, Oklahoma City, Oklahoma 73104. whereas in the $A / B$ study, anxiety (assumed to be characteristic of Type A personality) reduced differences. Obviously, it is impossible to compare normals, anxiety patients (under medication), and A/B type subjects on any strict set of criteria. Thus, conclusions regarding anxiety dimension in the success/failure paradigm are presently unclear. One way to shed some light on the issue, however, is to utilize normal subjects, selected for level of trait anxiety, which is a dimension likely to underlie the results of A/B type. Accordingly, the purpose of this experiment was to examine the role of anxiety in normal subjects within the success/ failure paradigm.

\section{METHOD}

\section{Subjects}

The subjects were 56 undergraduate students $(17$ males and 39 females) at the University of Oklahoma who participated for course credit. All subjects completed the Taylor MAS, along with a test battery to be used for other purposes (Meites, Lovallo, \& Pishkin, 1980).

\section{Test Scores}

The subjects were divided into high- and low-anxiety (MAS) groups according to the median, 23 above and 23 below the medial cutoff. One half of the subjects in each MAS group received feedback on Problem 1 that was consistently contingent on their responses; the other half were given $50 \%$ misinformative feedback and thus failed to solve. All subjects received contingent feedback on Problem 2.

\section{Tasks}

Each subject completed two CI tasks similar to those used by Pishkin and Shurley (1968). The tasks required the subjects to classify, by trial and error, geometric patterns based on three dimensions (size, shape, and color), each of which had two attributes or levels (large and small, square and circle, and red and blue, respectively). Classification was based on a conjunctive rule in which stimuli belonging to the concept contained both relevant attributes. The relevant attributes were different for the 
two problems. Feedback, whether veridical or misinformative, was provided on each trial. Testing proceeded until 16 consecutive correct responses were made or the subject reached Trial 80 , whichever came first.

\section{Apparatus}

The stimuli used for the CI problems were color slides, rear-projected onto a ground-glass screen. Subjects indicated their classification of each slide by pressing one of two buttons that advanced the slide projector and activated a light indicating the position of the correct response button for that trial. Feedback was provided by a pair of amber lights, one over each response button.

\section{Procedure}

Each subject was contacted by the experimenter and asked to volunteer for the study. Upon entering the laboratory, each subject was informed of the nature of the experiment, signed a consent form, and was instructed on the CI task, including practice utilizing the stimulus attributes. The two problems were presented with only a minimal pause between them, during which the experimenter changed slide trays and briefly redescribed the task to the subjects.

\section{RESULTS AND DISCUSSION}

A two-way analysis of variance was applied to the number of errors to criterion on the second CI problem, using high/low Taylor MAS scores and success/failure on the first CI problem as variables. The interaction of anxiety and success/failure was statistically significant $[F(1,35)=7.14, p<.01]$. These data are displayed in Figure 1. Following successful completion of the preliminary problem, subjects who were higher in anxiety performed better on Problem 2 than those who were lower in anxiety. Subjects exposed to misinformationinduced failure on Problem 1 performed well on Problem 2 if they were low in anxiety and poorly if they were high, although not subsantially worse than the subjects who had succeeded previously.

These data seem to suggest that prior failure increased

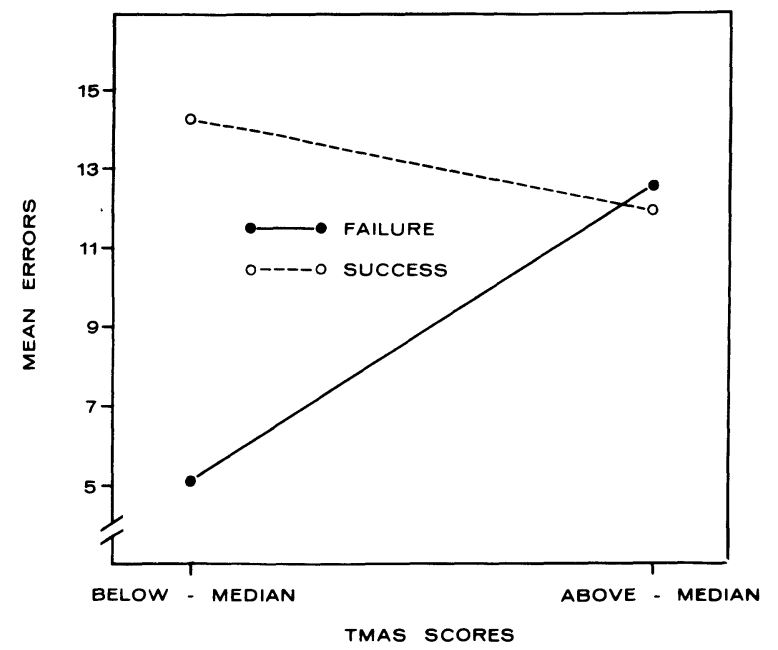

Figure 1. Mean errors as a function of anxiety and success/ failure experience.
Table 1

Mean Errors to Criterion in the Two Comparable CI Studies

\begin{tabular}{|c|c|c|c|c|}
\hline & \multicolumn{2}{|c|}{ This Study } & \multirow{2}{*}{\multicolumn{2}{|c|}{$\begin{array}{c}\text { Lovallo and } \\
\text { Pishkin (1980) }\end{array}$}} \\
\hline & \multirow{2}{*}{$\begin{array}{c}\text { High } \\
\text { Anxiety }\end{array}$} & \multirow{2}{*}{$\begin{array}{c}\text { Low } \\
\text { Anxiety }\end{array}$} & & \\
\hline & & & As & Bs \\
\hline Success & 12.4 & 13.5 & 46.4 & 32.8 \\
\hline Failure & 12.6 & 5.5 & 44.5 & 51.5 \\
\hline
\end{tabular}

the drive level of the low-anxiety subjects toward an optimal level. If this is the case, one of our series of studies with coronary-prone (Type A) and nonprone (Type B) young men (Lovallo \& Pishkin, 1980) produced effects opposite to the present data (i.e., lowanxiety, failure subjects of the present study were superior to the success subjects, whereas Type Bs showed reverse effect). It is probably safe to speculate that Type As, competitive, forceful, "speeded-up" individuals, fall in the above-median category on the Taylor MAS, with Bs falling below.

Table 1 summarizes findings from $\mathrm{A} / \mathrm{B}$ types, as well as findings from the present study. Comparison of the interactions of the A/B study (Lovallo \& Pishkin, 1980) and the present study reveal that As and high-anxiety samples do not differ as a result of success/failure manipulations. Bs show better performance after success than after failure; however, the low-anxiety group of this study was superior following failure. In short, Bs manifested cognitive deficit following failure, which is a finding consistent with previous studies utilizing normal (Pishkin, 1965) and psychiatric patient (Lovallo \& Pishkin, 1980; Pishkin \& Shurley, 1968) subjects. It is noteworthy that the Bs demonstrated opposite effects in CI performance when compared with low-anxiety subjects on the success/failure dimension of the present study. Thus, the usual characterization of coronaryprone behavior may not be related to anxiety parameters utilized in this experiment, and the anxiety component, as measured by the MAS, may not be essential in such a characterization. It must also be noted that problems employed in the study of $\mathrm{A} / \mathrm{B}$ personality types were more difficult than those of the present experiment. Conceivably, the interaction in question was influenced by the effects of problem difficulty. While that result seems unlikely in the light of other evidence (Pishkin, Fishkin, \& Shurley, 1979; Pishkin, Shurley, \& Wolfgang, 1967), it remains a possibility until it can be studied directly.

\section{REFERENCES}

Lovallo, W. R., \& Pishrin, V. Performance of Type A (coronary-prone) men during and after exposure to uncontrollable noise and task failure. Journal of Personality and Social Psychology, 1980, 38, 963-971.

Meites, K., Lovallo, W. R., \& Pishrin, V. A comparison of four scales of anxiety, depression and neuroticism. Journal of Clinical Psychology, 1980, 36, 427-432. 
Pishkin, V. Dimension availability with antecedent success or failure in concept identification. Psychonomic Science, 1965, 2, 69-70.

Pishkin, V., Fishkin, S. M., \& Shurley, J. T. Motivation in cognitive performance: Double-blind comparison of chlordiazepoxide with doxepin. Current Therapeutic Research, 1979, 25, 165-171.

Pishkin, V., Fishkin, S. M., Shurley, J. T., Lawrence, B. E., \& Lovallo, W. R. Cognitive and psychophysiologic response to doxepin and chlordiazepoxide. Comprehensive Psychiatry, 1978, 2, 171-178.
Pishkin, V., \& Shurley, J. T. Electrodermal and electromyographic parameters in concept identification. Psychophysiology, 1968, 5, 112-118.

Pishrin, V., Shurley, J. T., \& Wolfgang, A. Stress: Psychophysiological and cognitive indices in an acute double-blind study with hydroxyzine in psychiatric patients. Archives of General Psychiatry, 1967, 16, 471-478.

TAYLOR, J. Drive theory and manifest anxiety. Psychological Bulletin, 1956, 53, 243-244.

(Received for publication September 21, 1981.) 